\title{
Review: rivastigmine may improve cognitive outcomes in Alzheimer's disease
}

\author{
Birks J, Iakovidou V,Tsolaki M. Rivastigmine for Alzheimer's disease. Cochrane Review, latest version 26 May 1999. In: \\ Cochrane Library. Oxford: Update Software.
} QUESTION: In patients with Alzheimer's disease (AD), how safe and effective is
rivastigmine in improving cognitive outcomes?

\begin{abstract}
Data sources
Studies were identified by searching the Cochrane Controlled Trials Register, the Cochrane Dementia Group register of clinical trials, Medline, EMBASE/ Excerpta Medica, PsycLIT, CINAHL, and SIGLE; handsearching geriatric and dementia journals and conference abstracts; contacting pharmaceutical companies; and reviewing references of identified studies.
\end{abstract}

Studies were selected if they were randomised, concealed, double blind, placebo controlled trials of mine was given for $\geqslant 2$ weeks.

\section{Data extraction}

Data were extracted on methodological quality including concealment, blinding, intention to treat analysis, and patient follow up. Data on outcome measures were sought for individual patients. The outcomes presented here are improvement in cognitive function $(\geqslant 4$ points improvement on the cognitive subscale of the Alzheimer's Disease Assessment Scale [ADAS-Cog]), global clinical change (Clinician's Interview Based Impression of Change scale), and acceptability of treatment (drop out from the study), all at 26 weeks of follow up.

\section{Main results}

7 trials (3370 patients) were included. 4 phase III trials (2804 patients) assessed outcomes at 26 weeks, but 2 trials were unpublished and data from these were unavailable for this review. Some evidence existed that rivastigmine improved cognitive function. However, the unpublished trials included $49 \%$ of the phase III trial patients and their exclusion was likely to bias the overall results. The results of the meta-analysis are therefore unreliable. Of the 2 published phase III trials, 1 showed a statistically significant improvement on the ADASCog with both lower dose rivastigmine (odds ratio [OR] $0.46,95 \%$ CI 0.24 to 0.90 ) and higher dose rivastigmine (OR 0.32, CI 0.18 to 0.59 ). 1 trial of lower dose rivastigmine showed an improvement in global clinical state (OR 0.58 , CI 0.36 to 0.93 ) and 1 trial of higher dose rivastigmine showed a marginal improvement (OR 0.65 , CI 0.42 to 0.99 ). Withdrawals before the end of treatment did not differ between lower dose rivastigmine and placebo; both higher dose trials showed more withdrawals in rivastigmine recipients.

\section{Study selection} patients with probable $\mathrm{AD}$ and treatment with rivastig-

\section{Conclusions}

In patients with Alzheimer's disease, evidence of the benefit of rivastigmine is inconclusive. 1 of 2 published trials shows improvement in cognitive outcomes. Publication of the results of 2 phase III trials will enable stronger evidence through a meta-analysis.

\section{COMMENTARY}

The review by Birks $e t$ al is a rare example of an independent analysis of a drug development programme. The authors used careful, modern methodology, and the included trials used recognised outcome measures in appropriate patient groups. The results of the meta-analysis should therefore be valid. On the basis of trials, rivastigmine has been approved by regulatory authorities in many countries. Perhaps surprisingly, however, clinicians need to be cautious in their interpretation of these rivastigmine data. What has gone wrong?

The main problem is that the data for 2 large trials of rivastigmine, including those for almost half of the patients in the phase III trials, were not available for this review. Obviously, these missing data could have a major effect on the result of the quantitative analysis. As they stand, the results are liable to an unknown degree of bias and must be considered to be unreliable. It is unknown if the data from these missing trials were made available to the Federal Drug Administration and other regulatory authorities when they were assessing this drug.

This raises 2 important issues. Firstly, drug companies should be urged to release all the trial data on their drugs. ${ }^{12}$ Increasingly, as clinicians try to base their treatment decisions on unbiased information, they will be reluctant to use treatments where there appear to be gaps in the available evidence. Secondly, reviewers need to clearly report how much existing trial data were unavailable for the review-and the current reviewers are to be commended on this point. However, when a high proportion of existing data are unavailable, and when this is likely to produce a biased estimate of the effectiveness of the treatment (as is possibly the case here), then reviewers should hesitate before undertaking a statistical meta-analysis.

As this evidence currently stands, clinicians using the Cochrane Library to inform their decisions are likely to prefer alternative anticholinesterases to rivastigmine.

Roger Bullock, MA, MBBS, MRCPsych

Victoria Hospital Swindon, UK

For correspondence: Mrs J Birks, Medical Statistician,

Department of Geratology, University of Oxford, Oxford OX2 6HE, UK. Fax +44 (0)1865224108.
Roberts I. An amnesty for unpublished trials. One year on, many trials are unregistered and the amnesty remains open. BMJ 1998;317:763-4.

2 Sykes R. Being a modern pharmaceutical company: involves making information available on clinical trial programmes. BMJ 1998;317:1172. 